\title{
Avaliação de desvio dimensional em Inconel 718 após a retificação em diferentes técnicas de lubri-refrigeração
}

\author{
Evaluation of dimensional deviation in nickel-base, \\ Inconel 718, alloy after grinding under different \\ cooling-lubrication techniques
}

\author{
Déborah de Oliveira ${ }^{1}$, Rosemar Batista da Silva ${ }^{1}$ e
} Rosenda Valdés Arencibia ${ }^{1}$

\footnotetext{
${ }^{1}$ Laboratório de Ensino e Pesquisa em Usinagem - LEPU - FEMEC/UFU CP: 38400-902, Uberlândia, MG, Brasil e-mail: deborahrpoliveira@gmail.com,rosemar.silva@ufu.br, rosenda.arencibia@ufu.br
}

\section{RESUMO}

O Inconel 718 é uma liga à base de níquel que possui elevada resistência à fadiga e à fluência, ductilidade, rigidez, inércia química e capacidade de operar em elevadas temperaturas, por isto é bastante utilizada na fabricação de peças aeronáuticas de alto rendimento. Estas peças exigem tolerâncias dimensionais e geométricas estreitas que em geral podem ser conseguidas por meio do processo de retificação. No entanto este é um material de baixa usinabilidade, principalmente por ser bem suscetível aos danos térmicos que ocorrem nas peças usinadas durante a retificação. Neste sentido, ambas as funções de refrigeração e lubrificação dos fluidos de corte devem predominar no processo de retificação desta liga, logo qualquer tentativa para melhorar estas propriedades deles que podem representar um grande ganho. Somado a isso ainda é escassa a literatura específica de trabalhos que abordem o desvio dimensional de peças retificadas e como ele é afetado pelos parâmetros de corte. Neste sentido, este trabalho tem como objetivo contribuir com conhecimentos na área de retificação avaliando a influência de diferentes atmosferas lubri-refrigerantes no desvio dimensional na altura das peças em Inconel 718 retificadas. Foram testadas as atmosferas de usinagem, a seco e com fluido de corte, sendo que para esta a última o fluido de corte foi empregado em abundancia e também via a técnica da Mínima Quantidade de Lubrificante (MQL). Como parâmetros de corte foram testados dois valores de penetrações de trabalho, $20 \mu \mathrm{m}$ e $40 \mu \mathrm{m}$. Os resultados mostraram que os menores valores de penetração de trabalho geraram os menores desvios dimensionais, como seria esperado. Além disso, a técnica MQL se mostrou mais vantajosa para redução de desvio dimensional em relação às outras atmosferas de lubrirefrigeração testadas. Os desvios obtidos enquadram-se na faixa de tolerância qualidade de trabalho, IT5 a IT6, coincidindo com a faixa normalmente relatada na literatura específica para peças fabricadas em Inconel 718.

Palavras-chave: Retificação, Inconel 718, técnica MQL, Desvio Dimensional, Grau de Tolerância IT.

\begin{abstract}
Inconel 718 is a nickel based alloy that has high resistance to fatigue and creep, ductility, stiffness, chemical inertia and the ability to operate at high temperatures. These properties make the Inconel 718 attractive to be employed in high performance parts. These parts require narrow dimensional and geometric tolerances that can usually be achieved by the grinding process. However, this material presents low machinability, especially because its susceptibility to thermal damages during grinding. In this sense, both lubrication and cooling properties of the cutting fluid must predominate during grinding of this alloy, so any attempt to improve the cutting fluids properties will represent a great contribution. Also, specific literature is still scarce in scientific studies about evaluation of dimensional deviation of ground workpieces and how they are affected by the cutting parameters. In this sense, this work aims to contribute with knowledge in the grinding field by evaluating the influence of different cooling-lubrication techniques on the dimensional deviation of the Inconel 718 workpieces. The machining atmospheres tested were: dry and with cutting fluid. For the latter, cutting
\end{abstract}


fluid was applied via conventional coolant technique (flood) and via the Minimum Quantity Lubrication (MQL) technique. Two values of depth of cut $20 \mu \mathrm{m}$ and $40 \mu \mathrm{m}$ were testes as input parameters. The results showed that the lowest values of depth of cut $(20 \mu \mathrm{m})$ generated smaller dimensional deviations, as would be expected. In addition, grinding with the MQL technique proved to be advantageous in generating the lowest dimensional deviation values. After measurements of the dimensional deviations, IT tolerance grades were calculated and they were within the IT6 - IT5 range, which is in agreement with the one usually mentioned in the specific literature for parts manufactured in nickel-base, Inconel 718, alloy.

Keywords: Grinding, Inconel 718, MQL technique, Dimensional Deviation, IT Tolerance Grade.

\section{INTRODUÇÃO}

O desenvolvimento dos materiais metálicos está intimamente associado aos avanços tecnológicos e, para a grande maioria das aplicações em engenharia, os aços são os mais requisitados. Contudo, para algumas aplicações os aços não conseguem atender as exigências de projeto, por exemplo, de manter a resistência mecânica a elevadas temperaturas. E esta limitação dos aços torna ligas refratárias ou "superligas" à base níquel e de titânio, os preferíveis, já que elas reúnem a combinação de boas propriedades químicas e mecânicas que são mantidas tanto a elevadas temperaturas quanto em temperaturas criogênicas. As superligas à base de níquel se destacam pela combinação de boa resistência à fadiga e à fluência, resistência à corrosão em ambientes hostis, bem como ductilidade e rigidez $[1,2,3]$. Estas propriedades fazem as ligas à base de níquel serem atrativas para aplicações da indústria aeronáutica e do setor petrolífero.

Uma das principais ligas de níquel é o Inconel 718 para o qual as aplicações estendem desde criogenia, indústrias automobilística, petrolífera e naval, e principalmente aeroespacial. Em especial, o Inconel 718 apresenta capacidade de operar em elevadas temperaturas, segundo EZUGWU et al. [4] a fase de sua matriz (gama) possui estabilidade térmica até $900{ }^{\circ} \mathrm{C}$, elevada resistência mecânica e inercia química. Devido a estas propriedades, ele é muito empregado em peças críticas que precisam ser livres de defeitos e requerem bom acabamento e tolerâncias dimensionais que podem ser proporcionadas pelo processo de retificação, que é um processo capaz de garantir esta combinação. A Figura 1 mostra detalhes da raiz de diferentes pás de turbinas aeronáuticas $[5,6,7]$.
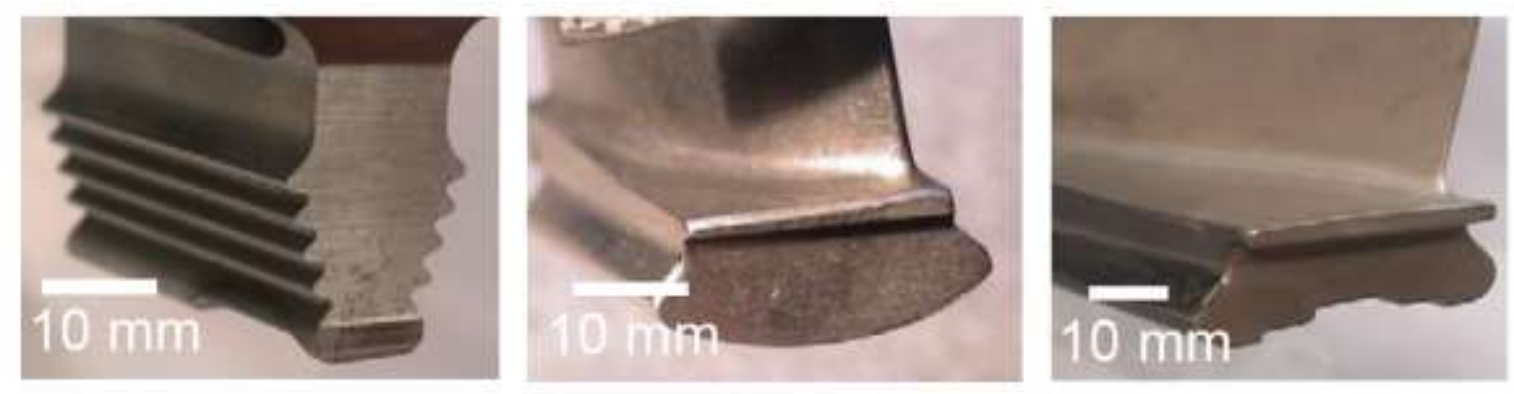

Figura 1: Detalhes da raiz de pás de turbina de motor aeronáutico (adaptado de ASPINWALL et al., 2007).

Entretanto, para que a qualidade final seja atendida, é preciso uma grande atenção na usinagem destas ligas, já que as mesmas propriedades, que são desejáveis para a peça, tornam-se desafios durante a usinagem como tem sido relatado na literatura. Este fato pode ser justificado, principalmente porque esta liga, além de apresentar elevada resistência mecânica, possui baixa condutividade térmica, o que implica no desenvolvimento de elevada geração de calor e de temperaturas na interface peça-ferramenta mais altas que aquelas observadas na usinagem de aços [4], que no caso de operação de usinagem com ferramenta de geometria definida representa maiores forças de corte, maior taxa de desgaste da ferramenta. Com isso, a usinabilidade do Inconel 718 é inferior àquela dos aços comuns ao carbono, por exemplo. E ao considerar o processo de retificação desta liga, a usinagem torna-se mais complexa por causa da natureza dos materiais abrasivos que compõem os rebolos e pelas características peculiares da formação do cavaco. Uma vez que tanto os rebolos abrasivos convencionais quanto ao Inconel em questão são pobres condutores de calor. Além disso, o Inconel 718 apresenta elevada resistência mecânica mesmo em altas temperaturas, o que o torna mais difícil de ser usinado. Quanto ao processo de retificação, é um processo que apresenta elevada energia específica, o que gera grandes quantidades de calor, durante a retificação a peça está propensa a ocorrência de danos térmicos tais como queima de retifica, trincas, tensão residual de tração, distorção microestrutural e dimensional, como 
também irregularidades superficiais que comprometem a sua funcionalidade e geram prejuízos $[8,9,10]$.

Uma das principais formas de reduzir esses danos de origem térmica é empregar fluidos de corte que possuam as funções de refrigeração para remover o calor gerado na interface ferramenta-peça e de lubrificação para reduzir o atrito entre os grãos abrasivos e a peça. E a técnica mais empregada em processos de retificação é a chamada convencional, ou abundância, em que o fluido de corte é aplicado em grandes vazões, superiores a $180 \mathrm{l} / \mathrm{h}(180000 \mathrm{ml} / \mathrm{h})[11,12]$.

Contudo, o emprego desta técnica vem perdendo espaço, pois alguns fluidos de corte podem causar danos ao operador e ao meio ambiente, além de serem responsáveis por cerca de $16 \%$ dos custos de produção de uma peça retificada. Desta forma, vários têm sido os esforços acadêmicos e industriais no sentido de aprimorar as técnicas de aplicação de fluido de corte já existentes como também de desenvolver uma nova técnica que permita não utilizar, ou minimizar o uso dos fluidos de corte [12].

E como alternativa, a técnica da Mínima Quantidade de Lubrificante (MQL) tem sido uma grande aliada na redução de volumes de fluidos de corte em usinagem. Nesta técnica, um baixo volume de óleo normalmente do tipo integral é aplicado em vazões inferiores a $500 \mathrm{~mL} / \mathrm{h}$ na zona de corte [13]. Além da vantagem do baixo volume de fluido de corte, a técnica alia o fato de utilizar um fluido com alta capacidade de lubrificação com o ar comprimido (em pressões em torno de $0,6 \mathrm{MPa}$ ) que tem a função de refrigeração da zona de corte. Outra vantagem, é que são dispensados os grandes reservatórios para armazenar o fluido de corte, além de exigir menor custo com manutenção e descarte dele. Com isso, é produzido um menor impacto ambiental e uma usinagem mais limpa em relação à técnica convencional de aplicação de fluido de corte, vantagens que fazem com que a sua aplicação seja cada vez mais priorizada em operações de usinagem $[11,14]$.

RABIEI et al. [15] investigaram a influência da atmosfera lubri-refrigerante nas forças do processo de retificação de quatro aços, CK45 (90 HRB), S305 (25 HRC), 100Cr6 (58 HRC) e HSS (62 HRC) com rebolo de alumina. As atmosferas testadas foram: convencional, a seco e MQL. Eles variaram ainda a penetração de trabalho em 5, 20, 35 e $50 \mu \mathrm{m}$ e como resultados observaram que a técnica MQL não apenas foi vantajosa para a redução na quantidade de fluido, como também melhorou a capacidade de lubrificação quando comparada com a técnica convencional para todos os aços. Isto ocorre, pois, esta técnica favorece a penetração do fluido na zona de contato, devido a maior pressão de aplicação do jato (0,6 MPa em média) em relação a técnica convencional, como também devido à maior velocidade do jato, permitindo assim que o fluido de corte ultrapasse com mais facilidade a camada de ar que se forma ao redor do rebolo, devido à rotação do rebolo e atinja assim alcance a região de corte. Além disso, estes autores observaram uma redução na força tangencial após a usinagem com a técnica convencional.

Mais recentemente, WANG et al. [16] também retificaram a liga de níquel GH4169 com rebolo de alumina (grana mesh 80) com diferentes tipos de fluidos de corte que foram aplicados pela técnica MQL. Eles testaram um único óleo mineral (parafina) e outros 7 óleos de origem vegetal (soja, amendoim, milho, colza, palma, mamona e girassol), além de um fluido sintético solúvel (CCF-04T), na concentração de $4 \%$ em água. Este último foi aplicado também via técnica convencional para fins de comparação. Estes autores relataram que a usinagem com a técnica MQL proporcionou os menores esforços de corte devido às suas propriedades lubrificantes e maior eficiência em garantir que o fluido alcance a zona de corte em relação à técnica convencional, independente do fluido de corte testado. Além disso, segundo os autores, os valores de rugosidade foram menores para as peças usinadas com óleos vegetais e que o menor valor $(\mathrm{Ra}=0,36 \mu \mathrm{m})$ foi registrado ao empregar o óleo de mamona. Por fim, os autores avaliaram as superfícies das amostras retificadas com um Microscópio Eletrônico de Varredura (MEV) e observaram sulcos mais proeminentes na amostra que foi usinada com o fluido sintético aplicado pela técnica convencional, enquanto que evidência de material aderido foi observada na amostra que foi usinada com óleo de soja aplicado via a técnica MQL.

Todos estes estudos visam melhorar a qualidade de peças retificadas, além de aprimorar o acabamento superficial e evitar danos térmicos na peça é necessário estar atento à obtenção da dimensão e da forma esperadas no projeto da peça, segundo norma A NBR ISO 2768-2 [17], a dimensão e a forma geométrica de uma peça devem vir acompanhadas de tolerâncias. Segundo AGOSTINHO et al. [18], os desenvolvimentos dos sistemas de tolerâncias estão intimamente ligados aos problemas de intercambialidade, possibilitando que o projetista coloque no desenho as dimensões limites, uma vez que se admite que nenhuma peça durante a fabricação estará isenta de alguma variação dimensional e/ou geométrica. A consideração principal na especificação de tolerâncias é a garantia da qualidade funcional da peça prevista em projeto. Ainda de acordo com estes autores, as dimensões de duas peças quaisquer de mesmo lote de peças fabricadas são diferentes, esta diferença é dada por diversos fatores, principalmente, a máquina-ferramenta, a fixação da peça, a localização da peça, o sistema de fixação da ferramenta, as peças em bruto e o ambiente de trabalho. 
De acordo com a NBR ISO 6158 [19], "uma tolerância de dimensão linear controla somente a dimensão efetiva local (medição entre dois pontos) de um elemento, mas com seus desvios de forma (por exemplo: desvios de circularidade e retitude de um elemento cilíndrico ou desvio de planeza de superfícies paralelas). E é importante ressaltar que não existe controle da inter-relação geométrica de elementos isolados pelas tolerâncias dimensionais". Esta norma ainda aborda o sistema de ajustes e tolerâncias, em que é definida a tolerância como a diferença entre a dimensão máxima e a dimensão mínima de um valor nominal, ou seja, diferença entre o afastamento superior e o afastamento inferior de uma dimensão. Além disso, a tolerânciapadrão ou da sigla da terminologia Inglesa International Tolerance (IT), que é tolerância internacional ou grau de tolerância-padrão, que é qualquer tolerância pertencente a este sistema. Os graus de tolerância-padrão correspondem ao mesmo nível de precisão para todas as dimensões nominais $[17,19]$ e em geral variam na faixa de IT0 a IT16 e dependem da dimensão nominal da peça, onde IT16 significa aquela tolerância mais aberta. Na Tabela 1 são apresentados parte dos valores numéricos de graus de tolerância-padrão IT para alguns grupos de dimensões nominais.

Tabela 1: Valores numéricos de graus de tolerância-padrão IT para dimensões nominais, (adaptada de [19]).

\begin{tabular}{|c|c|c|c|c|c|c|c|c|}
\hline \multirow{2}{*}{\multicolumn{2}{|c|}{ DIMENSÃO NOMINAL (MM) }} & \multicolumn{7}{|c|}{ GRAU DE TOLERÁNCIA PADRÃO } \\
\hline & & IT1 & IT2 & IT3 & IT4 & IT5 & IT6 & IT7 \\
\hline Acima & Até e inclusive & \multicolumn{7}{|c|}{ Tolerância $(\mu \mathrm{m})$} \\
\hline 3 & 6 & 1 & 1,5 & 2,5 & 4 & 5 & 8 & 12 \\
\hline 6 & 10 & 1 & 1,5 & 2,5 & 4 & 6 & 9 & 15 \\
\hline 10 & 18 & 1,2 & 2 & 3 & 5 & 8 & 11 & 18 \\
\hline 18 & 30 & 1,5 & 2,5 & 4 & 6 & 9 & 13 & 21 \\
\hline 30 & 50 & 1,5 & 2,5 & 4 & 7 & 11 & 16 & 25 \\
\hline
\end{tabular}

Para determinar o grau de tolerâncias padrão de peças que são retificadas mais especificamente por processo de retificação plana é preciso inicialmente calcular o valor de penetração de trabalho real ou efetivo. Sabe-se que no processo de retificação plana tangencial o rebolo penetra na peça uma quantidade prédeterminada e ajustada no anel graduado da retificadora, responsável pelo movimento vertical do rebolo, e conhecida como penetração de trabalho radial (ae). Contudo, devido à deformação de várias peças do sistema máquina-rebolo-peça, ao desgaste do rebolo e variações, dentre outros, a penetração de trabalho real do rebolo na peça (a) será inferior àquela ajustada (ae) [20]. Assim, de acordo com MARINESCU et al. [20] o valor da penetração de trabalho real ou efetiva pode ser estimado pela Eq. (1), que depende dos parâmetros que serão definidos na sequência:

$\mathrm{a}_{\mathrm{r}}=\mathrm{a}_{\mathrm{e}}-\delta-\mathrm{asw}+\mathrm{at}(\mu \mathrm{m})$

Em que: $\left(a_{\mathrm{r}}\right)$ é o valor real da penetração de trabalho, $\left(\mathrm{a}_{\mathrm{e}}\right)$ o valor da penetração de trabalho selecionado e ajustado no anel graduado do eixo $\mathrm{Z}$ máquina-ferramenta, $\delta$ é deflexão do rebolo-peça, asw é o desgaste do rebolo por passe e at diz respeito à expansão térmica da peça.

$\mathrm{Na}$ prática, a forma de obter o valor de material real removido com maior precisão é medindo-se a peça antes e depois do processo de retificação. Este valor, no caso de uma superfície plana é o desvio dimensional na altura da peça. E embora o procedimento possa parecer simples, trabalhos publicados sobre avaliação de desvio dimensional em peças retificadas, principalmente de Inconel 718 após a retificação, são escassos. Um dos trabalhos encontrados foi aquele produzido por TSO [21], quem testou dois tipos de rebolos (CBN e de alumina) no processo de retificação de Inconel 718 em diferentes condições de corte, e observou que a usinagem com o rebolo de CBN gerou o menor desvio dimensional nas peças em relação ao rebolo de alumina e que o procedimento em geral adotado de realizar o centelhamento do rebolo nos estágios iniciais do corte (spark in) também contribuiu para a redução deste desvio.

É possível perceber então que há a necessidade de controlar o processo a fim de se obter peças com baixos desvios dimensionais e geométricos. Uma aplicação que solicita tanto peças sem danos quanto com alta exatidão das dimensões é no setor aeronáutico, onde peças de Inconel 718 são comumente empregadas nas turbinas e partes que trabalham em elevadas temperaturas (partes quentes) dos motores aeronáuticos $[22,7,6]$.

ASPINWALL et al. [6] relataram que, apesar dos diversos estudos sobre os chamados blisk (do Inglês cuja união das palavras blade, que significa pá, e disk, que é disco) a grande maioria das turbinas dos motores aeronáuticos ainda utiliza pás intercambiáveis, que são usualmente fabricadas de ligas de níquel, tanto na 
turbina quanto nos estágios de alta compressão para alguns motores, pela vantagem de serem montadas no disco e pelo fato de permitir manutenção ou substituição apenas das pás danificadas, já que a usinagem normalmente em 5 eixos de uma peça bruta pode ser mais complexa e elevar o preço final. Na fabricação estas pás requerem acabamento e tolerâncias dimensionais que são proporcionadas pelo processo de retificação.

De acordo com KLOCKE et al. [22], os exemplos de pás de turbina apresentados por ASPINWALL et al [6], fabricados em Inconel 718 podem ser fabricados também pelo processo de usinagem por eletroerosão a fio e que proporcionam tolerâncias geométricas de até $5 \mu \mathrm{m}$. Para peças de turbinas a gás de motores, o processo de retificação ainda é um aquele mais empregado, pois às vezes são necessárias tolerâncias dimensionais inferiores a $10 \mu \mathrm{m}$ [7] e que implicam em tolerância de trabalho em torno de IT5.

Como a dimensão da raiz da pá, parte que também necessita do processo de retificação, varia entre 10 $\mathrm{mm}$ e $30 \mathrm{~mm}$ para as variações citadas por KLOCKE et al. [22], KLOCKE et al.[7] e ASPINWALL et al. [6], a tolerância de trabalho (IT) esperada para estes materiais situa-se na faixa entre IT6 e IT4. Esta tolerância pode ser convertida para qualquer dimensão adotada para o corpo de prova tomando como referência a tabela da NBR ISO 6158 [19] recomendada para esta finalidade. E foi o fato das tolerâncias para a fabricação deste tipo de peças serem estreitas, que motivou esta pesquisa, uma vez que a literatura específica não apresenta dados experimentais de controle dimensional em peças retificadas.

\section{MATERIAIS E MÉTODOS}

Para realização dos ensaios de retificação foram utilizadas amostras de Inconel 718 com dimensões de $15 \mathrm{~mm}$ de largura, $15 \mathrm{~mm}$ de altura e $20 \mathrm{~mm}$ de comprimento, fixadas em morsa de precisão, conforme Fig. 2.

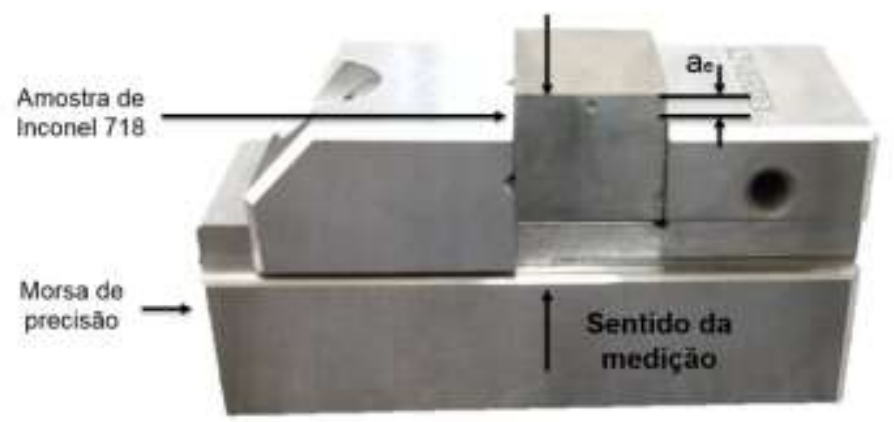

Figura 2: Montagem da peça de Inconel 718 na morsa de precisão com a indicação da penetração de trabalho (ae) e sentido da medição do desvio dimensional.

Os ensaios de retificação foram realizados em uma retificadora plana tangencial, do fabricante MELLO, modelo P-36, semiautomática, com potência nominal do motor que aciona o eixo-arvore de $2,25 \mathrm{~kW}$ e rotação constante em $2400 \mathrm{rpm}$. Esta máquina possui resolução de $5 \mu \mathrm{m}$ no anel graduado do eixo que executa o movimento vertical do rebolo (eixo $Z$ ) e erro não cumulativo de $\pm 2 \mu \mathrm{m}$ sobre o valor ajustado. Este erro médio do deslocamento do eixo $\mathrm{Z}$ foi obtido para este trabalho após a realização de 80 medições.

Nos experimentos foi empregado um rebolo de carbeto de silício (SiC), que é adequado para ligas à base de níquel e à base de titânio, vidro, ferros fundidos e afiação de ferramentas de metal duro [9, 23], com a seguinte especificação: 39C60KVK; do fabricante NORTON - SAINT GOBAIN ABRASIVES. Este rebolo possui ligante vitrificado com diâmetro externo de $305 \mathrm{~mm}$ por $25 \mathrm{~mm}$ de largura por $76 \mathrm{~mm}$ de diâmetro interno.

Foram utilizadas três atmosferas lubri-refrigerantes denominadas: seco (ou sem fluido), convencional e MQL. Para a atmosfera "convencional" utilizou-se um bocal de saída convencional na forma de sapata que acompanha a máquina ferramenta em que a vazão medida foi de 450 1/h $(450000 \mathrm{~mL} / \mathrm{h})$ com pressão de descarga próxima à pressão atmosférica. O fluido de corte é do tipo semi-sintético, emulsionavel Vasco 7000, com diluição de 1:9 em água, do fabricante Blaser Swisslube. Para a atmosfera lubri-refrigerante MQL foi utilizado o mesmo fluido de corte da técnica convencional, mas sem diluição em de água, e que foi aplicado à uma vazão de $240 \mathrm{ml} / \mathrm{h}$, com pressão de 0,4 Mpa, uma vez que para a aplicação de fluido ser considerada MQL a vazão deve ser inferior a $240 \mathrm{ml} / \mathrm{h}$ [13]. Na Figura 3 é apresentada a montagem experimental para os 
ensaios de retificação com os bocais utilizados para as duas técnicas de aplicação de fluido: convencional e MQL. Ressalta-se que, embora aparentemente haja diferença entre os bocais, a geometria dos bocais é semelhante para evitar que efeitos aerodinâmicos interfiram nos resultados do trabalho.

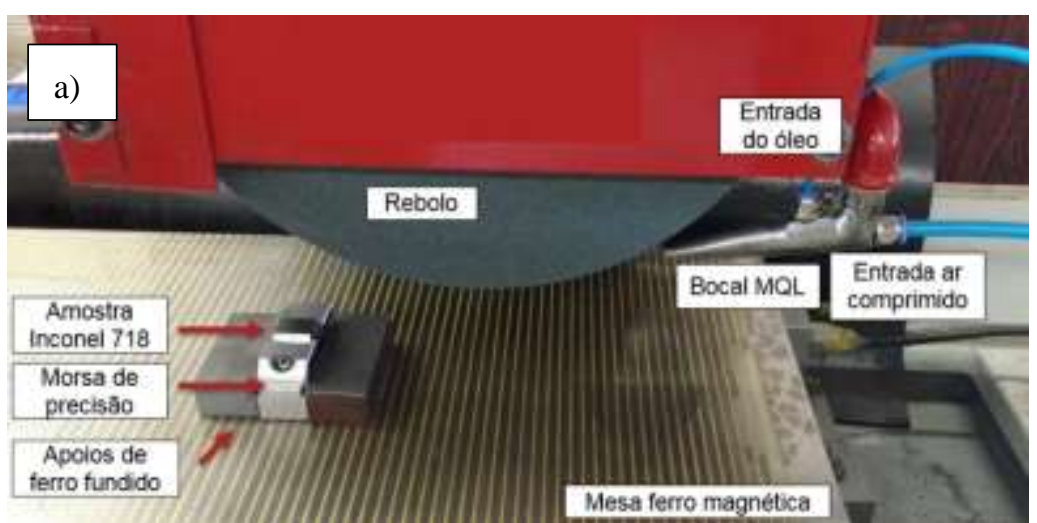

a) Montagem do bocal para usinagem com a técnica MQL.

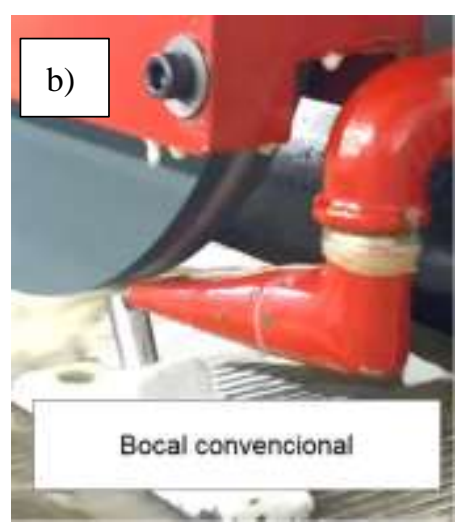

b) Detalhe do bocal convencional.

Figura 3: Bocais de aplicação do fluido de corte: a) Montagem do bocal para usinagem com a técnica MQL e b) Detalhe do bocal convencional

Com exceção da penetração de trabalho (ae), todos os outros parâmetros de corte, tais como a velocidade de corte e a velocidade da mesa, foram mantidos constantes. Apenas a penetração de trabalho radial foi variada. Nos ensaios experientais a velocidade transversal da mesa não foi variada, pois o rebolo percorreu toda a superfície da peça em um único passe, ou seja, $\mathrm{v}_{\mathrm{t}}=0 \mathrm{~m} / \mathrm{min}$. Assim a profundidade corte, ap, é a própria largura da peça e igual a $15 \mathrm{~mm}$. Na Tabela 2 é apresentado o resumo das condições utilizadas para o desenvolvimento desta pesquisa em processo de retificação plana do Inconel 718, estas condições foram escolhidas de acordo com a capacidade da retificadora plana tangencial e com base em outros trabalhos em retificação plana da mesma liga que foram desenvolvidos no Laboratório de Ensino e Pesquisa em Usinagem (LEPU) - FEMEC-UFU[24, 25, 26]. Ressalta-se ainda que estes valores de penetração de trabalho, como também da velocidades de corte e da mesa são comumente empregados para retificação de aços endurecidos, tais como aço SAE 52100 [27], por exemplo, outro material bastante susceptível a danos térmicos. Assim, os valores de $\mathrm{a}_{\mathrm{e}}=20 \mu \mathrm{m}$ e de $\mathrm{a}_{\mathrm{e}}=40 \mu \mathrm{m}$, representam condições mais branda (em que não presença de defeitos nas superfícies retificadas) e mais severa, e que há ocorrência de dano térmico.

Tabela 2:Variáveis de entrada utilizados nos ensaios de retificação.

\begin{tabular}{c|c}
\hline PARÂMETRO & VALOR \\
\hline \multirow{2}{*}{ Penetração de trabalho $-\mathrm{a}_{\mathrm{e}}(\mu \mathrm{m})$} & 20 \\
\cline { 2 - 2 } & 40 \\
\hline Velocidade de corte $\mathrm{v}_{\mathrm{s}}(\mathrm{m} / \mathrm{s})$ & 38 \\
\hline Velocidade da mesa $\mathrm{v}_{\mathrm{w}}(\mathrm{m} / \mathrm{min})$ & 10 \\
\hline Profundidade de corte $(\mathrm{mm})$ & 15 \\
\hline
\end{tabular}

Adotou-se como critério de parada dos ensaios de retificação com dois passes do rebolo por toda a superfície da peça para cada penetração de trabalho de forma que, para cada penetração de trabalho, $20 \mu \mathrm{m}$ e 40 $\mu \mathrm{m}$, foram realizados passes que resultam nos valores de remoção de material $6 \mathrm{~mm}^{3}$ e $12 \mathrm{~mm}^{3}$, respectivamente. Em todas as condições de ensaio foi realizada uma réplica.

Todos os ensaios de retificação (incluindo uma réplica para cada condição) foram realizados com a prévia dressagem do rebolo. Para isso, foi utilizado um dressador de ponta única de diamante (fabricante Winter) com raio de ponta de $0,3 \mathrm{~mm}$. A dressagem foi realizada nas mesmas condições empregadas para a usinagem com a técnica convencional de aplicação de fluido de corte. Foi adotado um grau de recobrimento do rebolo $(\mathrm{Ud})=3$, valor este que também tem sido empregado nos trabalhos desenvolvidos em retificação plana de aços endurecidos [27] e de Inconel 718 [24, 26]. O grau de recobrimento do rebolo, Ud, pode ser selecionado com os demais parâmetros de dressagem segundo a Eq. (2):

$U_{d}=\frac{b_{d}}{S_{d}}$ 
Onde bd: é largura de dressagem ou de atuação do dressador; e Sd o passo de dressagem.

O valor de Ud desta pesquisa foi selecionado por em geral ele variar entre 3 e 4 para processos de retificação de semi-precisão ou mediana [8], condição em que se enquadra esta pesquisa devido às condições de corte empregadas, principalmente pelas características do grão abrasivo, já que o rebolo possui grana mesh 60 , que resulta em um tamanho médio de grão abrasivo igual 0,250 mm, conforme informado previamente.

Para Ud $=3$ o valor de penetração de dressagem $(\mathrm{ad})$ inicialmente calculado foi de $10 \mu \mathrm{m}$. Este valor de ad foi ajustado conforme variação na ponta do diamante do dressador, que foi mantida sempre na mesma posição, (Eq. 2), considerando que o valor de bd deve-se manter constante a fim de garantir $\mathrm{Ud}=3$. Assim, quando o dressador tocava a superfície do rebolo, eram realizados dois passes completos para garantir uma dressagem uniforme. A cada operação de dressagem, a ponta do dressador era levada a um Estero microscópio SZ6145TR - OLYMPUS do LEPU para medição do seu desgaste. O parâmetro bd pode ser determinado pela Eq. (3).

$\mathrm{b}_{\mathrm{d}}=\sqrt{8 \times \mathrm{rpxad}}$

Onde:

rp é o raio de ponta do diamante do dressador.

ad: é a penetração de dressagem

O desvio dimensional foi medido na peça e na mesma direção da penetração de trabalho radial (ae) (Fig. 3), ou seja, na própria altura da peça. Ele representa a diferença entre o valor de penetração ajustado na máquina antes da retificação (valor esperado) e aquele de fato efetuado durante a usinagem, ou seja, o quanto de fato foi removido da peça durante a passagem do rebolo (valor resultante da passagem do rebolo), definido como $\mathrm{a}_{\mathrm{r}}$ neste trabalho, conforme o proposto na Eq. (1). Informações sobre análise deste desvio em retificação ainda são escassas na literatura, no entanto, sabe-se que existem deformações elásticas e dilatações térmicas no sistema máquina-rebolo-peça durante e após a usinagem da peça, fazendo com que a dimensão final da peça não seja exatamente aquela desejada, e por isso são estabelecidas tolerâncias dimensionais que variam conforme a peça e o processo de fabricação empregado.

Sumarizando, os parâmetros obtidos serão:

i. Diferença dimensional $(\mu \mathrm{m})$ : representa a quantidade linear (altura) de material removido da peça após a retificação, valor medido $\left(\mathrm{a}_{\mathrm{r}}\right)$.

ii. Desvio dimensional $(\mu \mathrm{m})$ : diferença entre a altura que se desejava remover, ou seja, a penetração de trabalho, e a realmente removida, valor calculado a partir da diferença dimensional e da penetração de trabalho.

iii. Tolerância de Trabalho, IT [19]: obtida a partir da norma, dimensão da peça e desvio dimensional.

Vale ressaltar que além dos fatores já citados a peça está sujeita a deformações plásticas, utilizando os valores de força obtidos por TSO [21] e a área de contato do rebolo com a peça durante a usinagem dada por MALKIN E GUO [9] é possível calcular a deformação elástica esperada na peça a partir das propriedades do material e da lei de Hooke. Da seguinte forma:

De acordo com MALKIN E GUO [9] a penetração do rebolo na peça resulta numa área de contato aparente onde a retificação de fato ocorre, e o comprimento $\left(l_{c}\right)$ do contato do rebolo com a peça é formado pelo arco AB na Figura 4. 


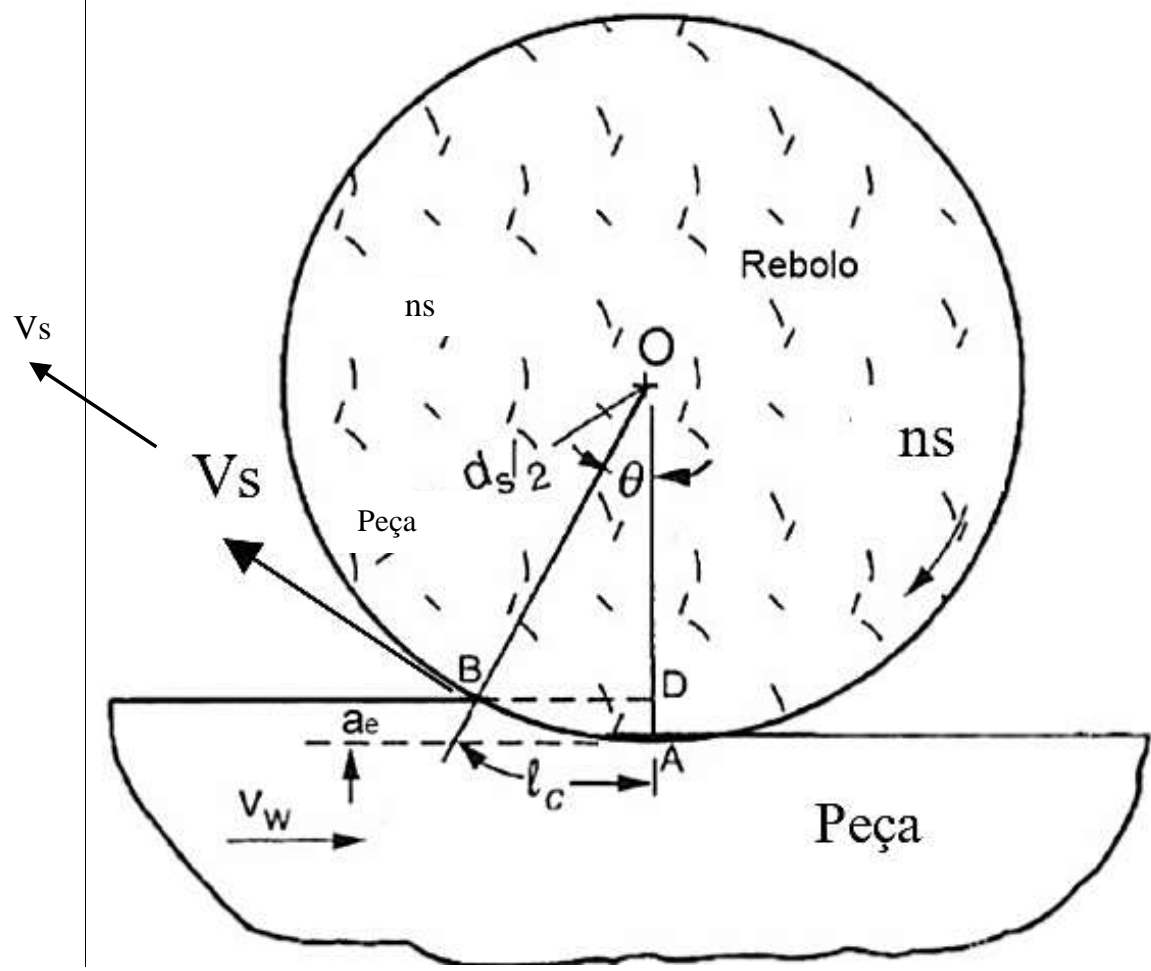

Figura 4: Comprimento de contato entre o rebolo e a peça (Adaptada de [9]).

De maneira geral $l_{c}$ é calculado pela Eq. (4):

$l_{c}=A B=\frac{d_{s} \theta}{2}$

$\mathrm{Na}$ Eq. (4) a área de contato $l_{c}$ pode ser obtida por uma relação entre o diâmetro equivalente do rebolo e o ângulo que representa a parte do rebolo em contato com a peça. Para retificação plana tangencial o ângulo $\theta$ pode ser determinado pela Eq. (5):

$\theta=\cos ^{-1}\left(1-\frac{2 a_{e}}{d_{s}}\right)$

Como $2 \mathrm{a}_{\mathrm{e}}<<<d_{s}$, a aproximação para ângulos pequenos pode ser feita, resultando na Eq. (6).

$\cos \theta=1-\frac{\theta^{2}}{2}$

Substituindo os dados da Eq. (6) na Eq. (4) obtém-se a Eq. (7).

$l_{c}=\left(a_{e} d_{s}\right)^{\frac{1}{2}}$

Sendo:

$\mathrm{a}_{\mathrm{e}}=$ penetração de trabalho

$d_{s}=$ diâmetro equivalente do rebolo (na retificação plana é o próprio diâmetro externo do rebolo)

Como $l_{c}$ é o arco AB, tem-se a Eq. (8):

$l_{c} l_{r}=a_{c e}$

Onde:

lr = largura rebolo

$a_{c e}=$ área de contato estimada

É importante determinar a área de contato do rebolo com a peça, pois através dela e das forças atuantes na retificação é possível identificar a pressão à qual a peça estará submetida. Considerando a pressão ho- 
mogênea na área, assim como a teoria de contato de Hertz obtém-se a Eq. (9), que permite calcular a pressão P.

$P=\frac{f}{l_{c} l_{r}}$

Da lei de Hooke tem-se a Eq. (10):

$P=\varepsilon E$

E que: $\varepsilon$ é a deformação

Substituindo a segunda parte da Eq. (10) na Eq. (11), é possível calcular a deformação da peça por meio da Eq. (11):

$\varepsilon=\frac{f}{l_{r}\left(a d_{S}\right)^{\frac{1}{2}} E}$

Por meio das equações anteriores e de dados da literatura é possível estimar a deformação elástica da peça durante o processo de retificação. Assim, para os valores de penetração de trabalho de $20 \mu \mathrm{m}$ e $40 \mu \mathrm{m}$ os valores os valores da deformação $(\varepsilon)$ esperados são $6,2 \mu \mathrm{m}$ e $8,7 \mu \mathrm{m}$, respectivamente.

A fim de minimizar erros no posicionamento da amostra na morsa devido às sucessivas retiradas da amostra, para medição deste desvio todas as amostras foram fixas em uma morsa de precisão (a mesma que é montada sobre a mesa ferromagnética da retificadora para os ensaios de retificação). Desta forma, o conjunto morsa-peça era levado até o Laboratório de Metrologia Dimensional da FEMEC-UFU para a medição do desvio dimensional e, para isso, foi utilizado um micrômetro para dimensões externas analógico do fabricante Mitutoyo com resolução de $1 \mu \mathrm{m}$ e faixa nominal de 25 a $50 \mathrm{~mm}$. O certificado de calibração do micrômetro, declara uma incerteza expandida associada à calibração igual a $2 \mu \mathrm{m}$, para um fator de abrangência $\mathrm{k}$ de 2,08 e probabilidade de abrangência de $95 \%$. Além disso, é declarada uma incerteza associada aos desvios de planeza das superfícies de medição fixa e móvel igual a $2 \mu \mathrm{m}$; e uma incerteza associada ao desvio de paralelismo entre as superfícies de medição no valor de $3 \mu \mathrm{m}$, todas com fator de abrangência $\mathrm{k}$ de 2,00 e probabilidade de abrangência de $95 \%$.

Como a morsa não tem a superfície de sua base contínua, foi utilizado também um bloco padrão de aço inoxidável com dimensão nominal de $5 \mathrm{~mm}$ para auxiliar nas medições. Todo o conjunto foi limpo com álcool etílico hidratado antes da realização das medições. Elas foram realizadas à temperatura de $20 \pm 1{ }^{\circ} \mathrm{C}$ para evitar erros ocasionados pela expansão térmica. Detalhes da montagem e da medição do desvio dimensional são apresentados na Fig. 4. Foram realizadas 10 medições em cada amostra antes e após os ensaios de retificação. Ao final, foi calculada a média aritmética dos valores indicados pelo micrômetro em cada condição, de forma que o desvio dimensional registrado é a diferença entre os valores de altura inicial da amostra e final medidos antes e após os ensaios de retificação, respectivamente.

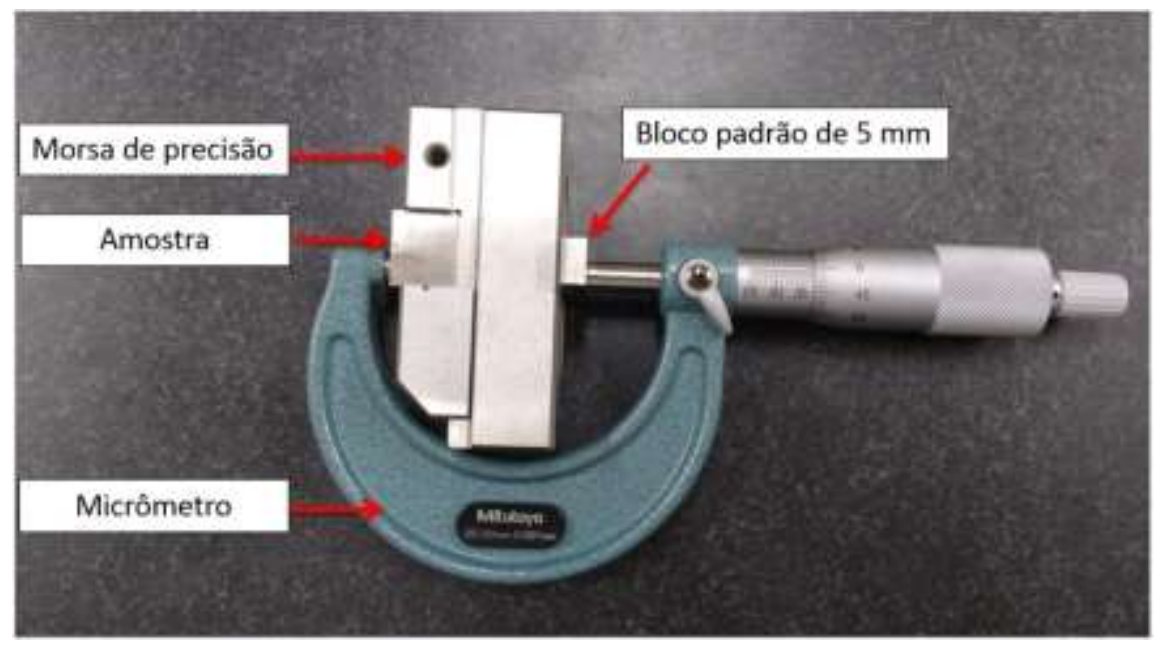

Figura 5: Montagem do conjunto morsa-peça-bloco padrão para a medição do desvio dimensional. 
Para monitoramento da temperatura foi utilizado um termo-higrômetro digital com resolução de 0,1 ${ }^{\circ} \mathrm{C}$ e faixa nominal de -20 a $60{ }^{\circ} \mathrm{C}$. O certificado de calibração do termo-higrômetro declara uma incerteza expandida associada à calibração de $0,3{ }^{\circ} \mathrm{C}$ para a faixa nominal de temperatura, com fator de abrangência $k$ igual a 2,00 e infinitos graus de liberdade.

\section{RESULTADOS E DISCUSSÕES}

$\mathrm{Na}$ Tabela 3 são apresentados os valores de diferença dimensional $(\mu \mathrm{m})$ e desvio dimensional $(\mu \mathrm{m})$ bem como as tolerâncias de Trabalho atribuídas às peças retificadas, que estão também plotados na forma de gráfico e apresentados na Figura 6. Ressalta-se que, ao observar esses resultados, deve-se atentar ao fato de que os valores de deformação ( $\varepsilon$ ) esperados é $6,2 \mu \mathrm{m}$ e de $8,7 \mu \mathrm{m}$ para os valores de penetração de trabalhos $20 \mu \mathrm{m}$ e $40 \mu \mathrm{m}$, respectivamente. Na Tabela 3 é ainda apresentado o valor do desvio padrão associado aos valores de diferença dimensional para uma confiabilidade de $95,45 \%$.

Tabela 3: Resultados dimensionais de amostras de Inconel 718 retificadas para as diferentes condições investigadas.

\begin{tabular}{|c|c|c|c|c|}
\hline $\begin{array}{l}\text { Penetração } \\
\text { de trabalho } \\
\left(\mathrm{a}_{\mathrm{e}-\mu \mathrm{m})}\right.\end{array}$ & $\begin{array}{l}\text { Atmosfera } \\
\text { lubri-refrigerante }\end{array}$ & $\begin{array}{l}\text { Diferença } \\
\text { Dimensional } \\
\left(\mathrm{a}_{\mathrm{r}}-\mu \mathrm{m}\right)\end{array}$ & $\begin{array}{l}\text { Desvio } \\
\text { Dimensional } \\
\left(\mathrm{a}_{\mathrm{e}}-\mathrm{a}_{\mathrm{r}}-\mu \mathrm{m}\right)\end{array}$ & $\begin{array}{l}\text { Tolerância de Trabalho, } \\
\text { IT (NBR ISO 6158) }\end{array}$ \\
\hline \multirow{3}{*}{20} & A Seco & $12 \pm 5$ & 8 & IT5 \\
\hline & Convencional & $10 \pm 2$ & 10 & IT6 \\
\hline & MQL & $11 \pm 1$ & 9 & IT6 \\
\hline \multirow{3}{*}{20 - Réplica } & A Seco & $10 \pm 3$ & 10 & IT6 \\
\hline & Convencional & $10 \pm 4$ & 10 & IT6 \\
\hline & MQL & $10 \pm 1$ & 10 & IT6 \\
\hline \multirow{3}{*}{40} & A Seco & $21 \pm 4$ & 19 & IT8 \\
\hline & Convencional & $12 \pm 2$ & 28 & IT9 \\
\hline & MQL & $26 \pm 1$ & 14 & IT7 \\
\hline \multirow{3}{*}{40 - Réplica } & A Seco & $19 \pm 3$ & 21 & IT8 \\
\hline & Convencional & $17 \pm 3$ & 23 & IT8 \\
\hline & MQL & $27 \pm 1$ & 13 & IT7 \\
\hline
\end{tabular}

Na Figura 6 é apresentada a diferença dimensional medida próxima à esperada $\left(\mathrm{a}_{\mathrm{e}}\right)$ para efeito de comparação com o desvio dimensional de fato medido nos ensaios. É possível observar que ao empregar o maior valor de penetração de trabalho obteve-se a maior diferença entre o valor esperado $\left(\mathrm{a}_{\mathrm{e}}\right.$ ajustado na máquina) e o medido $\left(\mathrm{a}_{\mathrm{r}}\right)$. E este resultado está de acordo com o proposto por MARINESCU et al. [20] que afirmaram que quanto maior o valor da penetração de trabalho maiores serão os esforços aos quais o conjunto máquina-ferramenta-peça será submetido e, portanto, maiores serão as deflexões do sistema. Com isso, era de se esperar que o maior valor de penetração de trabalho implicasse na maior diferença de material removido pelo rebolo. O desvio padrão (para uma confiabilidade de 95,45\%) é mostrado na Figura 6 como sendo a barra de erros. 


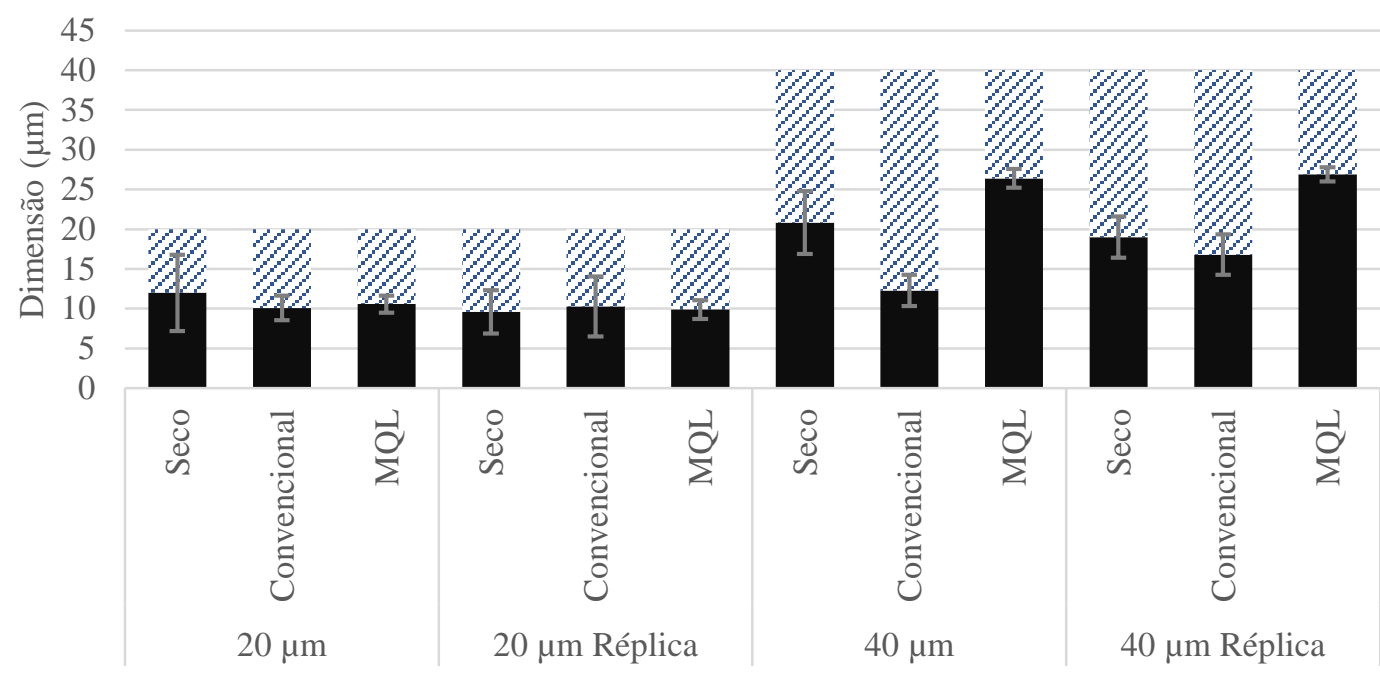

- Diferença dimensional $(\mathrm{ar}-\mu \mathrm{m}) \quad$ ¿ Desvio dimensional (ae-ar - $\mu \mathrm{m})$

Figura 6: Resultados da medição dimensional.

Além disso, o aumento do valor da penetração de trabalho pode influenciar no aumento do desgaste do rebolo, já que haverá uma maior área dos grãos em contato com o material da peça o que irá aumentar o atrito e, consequentemente, o desgaste. Em última instância poderá haver ainda a quebra e destacamento dos grãos do rebolo. Pode-se observar ainda da Tabela 3 que para os menores valores de penetração de trabalho, $\mathrm{a}_{\mathrm{e}}=20 \mu \mathrm{m}$, foi possível obter peças com tolerâncias dimensionais equivalentes àquelas propostas por KLOCKE et al. [22], KLOCKE et al. [7] e ASPINWALL et al. [6], IT6-IT5, que são tolerâncias consideradas estreitas e aplicadas para peças críticas fabricadas de Inconel 718.

Da Tabela 3 e Figura 6 pode-se inferir que a atmosfera lubri-refrigerante interfere na qualidade da peça, principalmente quando se empregam maiores valores de penetração de trabalho, enquanto que para o menor valor apenas são observadas tendências. A aplicação de fluido de corte via a técnica MQL resultou em menores desvios dimensionais na maioria dos casos e, consequentemente, na melhor qualidade IT das peças nas condições investigadas. Além disso, o baixo desvio padrão indica que o emprego desta técncia promove um processo com menor variação ou de melhor controle, conferindo ás peças retificadas nesta condição maior repetibilidade dimensional. Isso se deve à combinaçao da elevada capacidade de lubrificação do fluido de corte (apenas óleo) e função de refrigeração do ar comprimido da técnica MQL que reduz os esforços durante o processo, melhora as condições tribológicas e reflete positivamente nos menores desvios dimensionais [28].

Já a usinagem com a atmosfera com fluido aplicado pela técnica convencional não se apresentou tão eficiente para o processo em termos de minimização de desvios dimensionais do Inconel 718, o que pode estar associado a sua maior capacidade de refrigeração, já que o fluido de corte é aplicado em abundãncia, o que promove maior retirada de calor na interface rebolo-peça. Se por um lado a refrigeração eficiente previne a ocorrência de danos térmicos na peça, por outro lado ela confere maior resistência ao cisalhamento do material, o que eleva as forças de corte, principalmente daquelas as quais o conjunto máquina-ferramentapeça está submetido. Com isso, elevam-se as deflexões do sistema e, consequentemente, refletindo em maiores desvios na dimensão final da peça [20].

Quanto à atmosfera de usinagem a seco, ela resultou em desvios dimensionais baixos ao empregar condições mais brandas de usinagem. No entanto ao aumentar o valor da penetração de trabalho de $20 \mu \mathrm{m}$ para $\mathrm{a}_{\mathrm{e}}=40 \mu \mathrm{m}$, os resultados não foram satisfatórios, e a atmosfera que empregou a técnica MQL se mostrou mais eficiente. Este efeito pode ser explicado pelo termo at na Eq. (1) que diz respeito às variações de expansão térmica do material durante a usinagem, uma vez que o fluido de corte, além de remover calor e lubrificar a interface rebolo-peça, refrigera todo o corpo da peça usinada.

Ao observar atentamente os valores dos desvios obtidos para a menor penetração de trabalho, concluise que os valores da diferença dimensional variaram em torno de $10 \mu \mathrm{m}$, valor que parece baixo quando comparado com aquele esperado para a penetração de trabalho $\mathrm{a}_{\mathrm{e}}=20 \mu \mathrm{m}$. No entanto, o valor calculado para a diferença final, ao se descontar a deformação, é de 13,8 $\mu \mathrm{m}$. Além disso, pode-se adicionar a este valor 
também o erro de posição do eixo $\mathrm{Z}$ da retificadora, que, conforme comentado na metodologia deste trabalho, pode variar entre 0 e $2 \mu \mathrm{m}$, o que corrobora com a consistência da metodologia utilizada neste trabalho.

Com relação à maior penetração de trabalho $\mathrm{a}_{\mathrm{e}}=40 \mu \mathrm{m}$, é possível observar um comportamento diferente daquele encontrado para a penetração de trabalho de $20 \mu \mathrm{m}$. Ao empregar a técnica MQL, observa-se que ela foi novamente mais eficiente que a técnica convencional e a condição a seco em termos de menores desvios dimensionais. A remoção de material esperada seria igual ao $\mathrm{a}_{\mathrm{e}}$, considerando a deflexão de $8,7 \mu \mathrm{m}, \mathrm{o}$ valor de material removido deve ser de aproximadamente $31 \mu \mathrm{m} \pm 2 \mu \mathrm{m}$ (proveniente do erro da retificadora), a diferença dimensional foi de $27 \mu \mathrm{m} \pm 1 \mu \mathrm{m}$ para a réplica e $26 \mu \mathrm{m} \pm 1 \mu \mathrm{m}$ para o primeiro ensaio.

\section{CONCLUSÕES}

A partir do estudo de analise dimensional na altura de amostras de Inconel 718 após a retificação plana tangencial com rebolo de carbeto de silício realizado neste trabalho, as seguintes conclusões podem ser retiradas:

i. O emprego de menor valor de penetração de trabalho gera menores desvios dimensionais como esperado, mostrando grande influência das deformações elásticas na qualidade final da peça;

ii. A aplicação de fluido de corte via a técnica MQL resultou em menores desvios dimensionais, independente da penetração de trabalho empregada, portanto, ela apresentou desempenho superior que as demais atmosferas de usinagem empregadas neste trabalho;

iii. Atmosfera de usinagem a seco resultou em qualidades de trabalho IT5 e IT8 quando empregados o menor e o maior valor de ae, respectivamente. Já a técnica MQL resultou em qualidades de trabalho IT6 e IT7, e foi mais eficiente que a usinagem a seco em condições mais severas de retificação. O emprego da técnica convencional resultou em qualidades de trabalho bem semelhantes aquelas obtidas ao utilizar a técnica MQL, mas com a leve tendência de abertura de tolerância (IT9) ao empregar condição mais severa de retificação.

iv. No geral, este trabalho mostrou que é possível realizar a medição de desvio dimensional em peças retificadas. Além disso, os resultados obtidos se enquadraram dentro de tolerâncias equivalentes às aquelas relatadas na literatura (de IT4 a IT6) sobre peças de Inconel 718 submetidas ao processo de retificação, já que ao empregar a menor penetração de trabalho, ae $=20 \mu \mathrm{m}$, foram obtidas peças com qualidade de trabalho na faixa IT6- IT5, independentemente da técnica de lubri-refrigeração utilizada.

\section{AGRADECIMENTOS}

Os autores deste trabalho agradecem às empreas Saint Gobain Abrasivos da América do Sul e a Blaser SwissLube pela doação do rebolo e fluido de corte, respectivamente. O presente trabalho foi realizado com apoio da Coordenação de Aperfeiçoamento de Pessoal de Nível Supe-rior - Brasil (CAPES), Código de Financiamento 001. Eles agradecem ainda ao CNPq e FAPEMIG, ao Programa de Pós-Graduação em Engenharia Mêcanica, à Pró-Reitoria de Pesquisa e Pós-Gradução da UFU e a Faculdade de Engenharia Mecânica da UFU pelo apoio financeiro. O autor Rosemar Batista da Silva agradece em especial ao CNPq pelo apoio por meio de bolsa de Produtividade em Pesquisa - PQ - 2013, processo no. 308067/2013-4, já que este trabalho está relacionado ao projeto da bolsa.

\section{BIBLIOGRAFIA}

[1] ASM, Properties and selection - Nonferrous alloys and special-purpose materials, 10 ed., ASM International, 1990.

[2] EZUGWU, E.O., WANG, Z. M., MACHADO, A. R., "The machinability of Nickel-Based Alloys: A Review", Journal of Materials Processing Technology, v. 86, pp. 1-16, 1999. https://doi.org/10.1016/S09240136(98)00314-8

[3] DOS SANTOS, J.C., "Estudo do uso de ferramentas cerâmicas na usinagem de Inconel 718", 64 f., Dissertação de Mestrado, Faculdade de Engenharia do Campus de Guaratinguetá, Universidade estadual Paulista, Guaratinguetá, Guaratinguetá, São Paulo, Brasil, 2010.

[4] EZUGWU, E.O. O., BONNEY, J., YAMANE, Y., "An overview of the machinability of aeroengine alloys", Journal of Materials Technology. v. 134, pp.233 - 253, 2002. 
[5] CAMPBELL, F. C. Manufacturing Technology for Aerospace Structural Materials, 1 ed., Elsevier Science, 2006.

[6] ASPINWALL, D. K., SOO, S. L., CURTIS, D. T., MANTLE, A. L., "Profiled superabrasive grinding wheels for the machining of a nickel based superalloy", Annals of the CIRP, v. 56, pp. 335-338, 2007. https://doi.org/10.1016/j.cirp.2007.05.077

[7] KLOCKE, F., SOO, S. L., KARPUSCHEWSKI, B., WEBSTER, J. A., NOVOVIC, D., ELFIZY, A., AXINTE, D. A., TONISSEN, S., "Abrasive machining of advanced aerospace alloys and composites", CIRP Annals - Manufacturing Technology, pp. 581-604, 2015. https://doi.org/10.1016/j.cirp.2015.05.004

[8] MARINESCU, I. D.; HITCHINER, M.; UHLMANN, E.; ROWE, W. B.; INASAKI, I., Handbook of Machining with Grinding Wheels, New York: CRC Press, 2007.

[9] MALKIN, S.; GUO, C., Grinding technology: theory and application of machining with abrasives, 2.ed. New York: Industrial Press Inc., 2008.

[10] DEVILLEZ, A., LE COZ, G., DOMINIAK, S., DUDZINSKI, D., "Dry Machining of Inconel 718", Workpiece Surface Integrity. Journal of Materials Processing Technology, pp. 1590-1598, Mai. 2010. https://doi.org/10.1016/j.jmatprotec.2011.04.011

[11] IRANI, R.A., BAUER, R.J., WARKENTIN, A., "A review of cutting fluid application in the grinding process", International Journal of Machine Tools \& Manufacture, pp.1696-1705, Abr. 2005. https://doi.org/10.1016/j.ijmachtools.2005.03.006

[12] SANCHEZ, J.A., POMBO, I., ALBERDI, R., et al., "Machining evaluation of a hybrid MQL-CO2 grinding technology", Journal of Cleaner Production, pp. 1840-1849, Jul. 2010. https://doi.org/10.1016/j.jclepro.2010.07.002

[13] WALKER, T., The MQL Handbook, A guide to machining with minimum quantity lubrication, Unist, 2013.

[14] MARQUES, A., NARALA, S.K.R., MACHADO, A.R., et al., "Performance assessment of MSQL: Minimum quantity solid lubricant during turning of Inconel 718", Proc IMechE Part B: Journal Engineering Manufacture, pp. 1-16, Mai 2015. https://doi.org/10.1177/0954405415592128

[15] RABIEI, F., RAHIMI, A.R., HADAD, M.J., et al., "Performance improvement of minimum quantity lubrication (MQL) technique in surface grinding by modeling and optimization", Journal of Cleaner Production, pp. 447-460, 2015. https://doi.org/10.1016/j.jclepro.2014.08.045

[16] WANG, Y., LI C., ZHANG, Y., et al., "Experimental evaluation of the lubrication properties of the wheel/workpiece interface in minimum quantity lubrication (MQL) grinding using different types of vegetable oils", Journal of Cleaner Production 127, pp. 487-499, Jun. 2016. https://doi.org/10.1016/j.jclepro.2016.03.121

[17] NBR ISO 2768-2, Tolerâncias gerais - Parte 2: Tolerâncias geométricas para elementos sem indicação de tolerância individual, Rio de Janeiro, Rio de Janeiro, Brasil, 2001.

[18] AGOSTINHO, O L., RODRIGUES. A. C. S., LIRANI, J., Tolerâncias, ajustes, desvios e análise de dimensões, Edgard Blucher, 2004.

[19] NBR ISO 6158, Sistema de Tolerâncias e Ajustes, Rio de Janeiro, Rio de Janeiro, Brasil, 1995.

[20] MARINESCU, I. D., ROWE, W. B., DIMITROV, B., Tribology of abrasive machining processes, 1 ed., Willian Andrew, New York, 2004.

[21] TSO, P., "Study on the grinding of Inconel 718", Journal of Materials Processing Technology, p 421426, 1994. https://doi.org/10.1016/0924-0136(95)02026-8

[22] KLOCKE, F., KLINK, A., VESElOVAC, D., ASPINWALl, D.K., SOO, S. L., SCHMIDT, M., SCHILP, J., LEVY, G, KRUTH, J., "Turbomachinery component manufacture by application of electrochemical, electro-physical and photonic processes", CIRP Annals - Manufacturing Technology, pp. 703-726, 2014. https://doi.org/10.1016/j.cirp.2014.05.004

[23] MACHADO, A.R., ABRÃO, A. M., COELHO, R. T., DA SILVA, M. B. Teoria da usinagem dos materiais, 2 ed., Blucher, São Paulo, 2011.

[24] OLIVEIRA, D., Retificabilidade do Inconel 718 com base no acabamento e na microdureza, trabalho de conclusão de curso, Universidade Federal de Uberlândia, Uberlândia, Minas Gerais, Brasil, 2015.

[25] DA SILVA, R. B., OLIVEIRA, D., CASTRO, P. H. C., et al., "Grinding of Inconel 718 alloy with airoil-water mixture delivered by MQL technique", STLE - Annals, 2016. 
[26] DE OLIVEIRA, D. Retificação de Inconel 718 com Multicamadas de Grafeno Dispersas em Fluido de Corte Aplicado via Técnica MQL, Dissertação de Mestrado, Universidade Federal de Uberlândia, Minas Gerais, Brasil, 2017.

[27] DE PAIVA, R.L. Aplicação de fluido de corte a baixas temperaturas na retificação plana tangencial do aço SAE 52100, Dissertação de Mestrado, Universidade Federal de Uberlândia, Minas Gerais, Brasil, 2016.

[28] FRAGOSO, K. M., UMBERTO, D. R., BARILI, I. C., et al., "Retificação cilíndrica do aço VP50 utilizando o rebolo de carbeto de silício verde com a técnica de MQL”, Revista Matéria, pp.756-766, 2016.

\section{ORCID}

Déborah de Oliveira

https://orcid.org/0000-0002-7340-5792

Rosemar Batista da Silva

https://orcid.org/0000-0001-5746-3020

rosenda Valdés Arencibia

https://orcid.org/0000-0001-9543-8774 